\title{
Efficacy and Tolerability of Generic Mirtazapine (Mirtax) for Major Depressive Disorder: Multicenter, Open-label, Uncontrolled, Prospective Study
}

\author{
Hoo Rim Song, ${ }^{1,2}$, Won-Myong Bahk², Young Sup Woo², Jong-Hyun Jeong ${ }^{2}$, Young-Joon Kwon', Jeong Seok Seo ${ }^{3}$, \\ Won $\mathrm{Kim}^{4}$, Moon-Doo Kim ${ }^{5}$, Young-Chul Shin 6 , Sang-Yeol Lee, Kyung Joon Min \\ Department of Psychiatry, 'Soonchunhyang University Cheonan Hospital, Cheonan, ${ }^{2}$ College of Medicine, The Catholic University of Korea, \\ Seoul, ${ }^{3}$ Konkuk University Chungju Hospital, Chungju, ${ }^{4}$ Inje University Seoul Paik Hospital, Seoul, ${ }^{5}$ Jeju National University Hospital, Jeju, \\ ${ }^{6}$ Kangbuk Samsung Hospital, Seoul, ${ }^{7}$ Wonkwang University Hospital, Iksan, ${ }^{8}$ Chung-Ang University Hospital, Seoul, Korea
}

\begin{abstract}
Objective: Mirtax is a generic mirtazapine widely used since 2003. We conducted an open-label, uncontrolled 6-week study to evaluate the efficacy and safety of Mirtax for major depressive disorder (MDD).

Methods: Ninety three MDD patients with the diagnosis of MDD and 17-item Hamilton Depression Rating Scale (HDRS) Score $\geq 14$ were recruited. The HDRS, Montgomery-Åsberg Depression Rating Scale (MADRS), and the Clinical Global Impressions-Severity Scale (CGI-S) were administered at baseline, 1, 2, 4 and 6 weeks. Response ( $\geq 50 \%$ decrease in the HDRS or MADRS score), remission (absolute HDRS score $\leq 7$ or MADRS score $\leq 10$ ) and CGI-I score $\leq 2$ were also calculated. Adverse event (AE) frequency and severity, weight, blood pressure, and pulse rate were checked to assess safety.

Results: The starting dosage was $11.5 \pm 6.4 \mathrm{mg} /$ day, and the maintenance dosage was $23.1 \pm 9.4 \mathrm{mg} /$ day. During 6 weeks, HDRS,

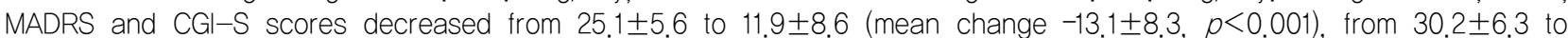
$13.73 \pm 10.40$ (mean change $-16.5 \pm 9.8, p<0.001$ ), and from $5.0 \pm 0.8$ to $2.5 \pm 1.3$ (mean change $-2.5 \pm 1.3, p<0.001$ ), respectively. The percentages of responders, remitters by HDRS and patients with a CGl-I score $\leq 2$ were $64.6 \%, 35.4 \%$ and $52.7 \%$, respectively. Significant decreases in HDRS, MADRS and CGI-S scores were confirmed at week 1. The total rate of AEs was 32.3\%; the most frequently reported AEs were sedation (4.3\%) and constipation (4.3\%). Weight was increased from 58.8 \pm 10.6 to $60.3 \pm 9.3 \mathrm{~kg}$ (mean change $0.7 \pm 1.7 \mathrm{~kg}, p=0.004$ ).

Conclusion: This study, as the first clinical trial of generic mirtazapine, demonstrated the efficacy and tolerability of Mirtax for MDD using a single treatment design.
\end{abstract}

KEY WORDS: Mirtazapine; Generic drugs; Efficacy; Tolerability.

\section{INTRODUCTION}

Depression is a common and longstanding illness, which contributes to the major global burden of disease. In 2009, the World Health Organization states that approximately 350 million people affected depression, and it is the second leading cause of disability worldwide. ${ }^{1)}$ Antidepressants can be an effective form of the treatment for major depressive disorder (MDD). Among them, mirtazapine is a widely used antidepressant due to its unique pharmacological profile and good psychiatric efficacy. It mainly

Received: October 30, 2014 / Revised: December 17, 2014 Accepted: December 22, 2014

Address for correspondence: Won-Myong Bahk, MD, PhD Department of Psychiatry, Yeouido St. Mary's Hospital, College of Medicine, The Catholic University of Korea, 10, 63-ro, Yeongdeungpogu, Seoul 07345, Korea

Tel: +82-2-3779-1051, Fax: +82-2-780-6577

E-mail:wmbahk@catholic.ac.kr blocks 5-hydroxytryptamine (5-HT) $)_{2}, 5-\mathrm{HT}_{3}$ and $\alpha_{2}$-auto and heteroreceptors, subsequently enhancing noradrenergic and serotonergic transmission. It also acts as a potent histamine receptor antagonist, which causes a sedative effect. ${ }^{2)}$ Mirtazapine showed good antidepressant efficacy equal to selective serotonin reuptake inhibitor $(\mathrm{SSRI})^{3)}$ or serotonin-norepinephrine reuptake inhibitor (SNRI), ${ }^{4)}$ and advantages especially in the early course of treatment. ${ }^{5}$

Since 1996, mirtazapine has been internationally available for the treatment of MDD, including in the United States. During this period, many generic forms of mirtazapine were released following bioequivalence tests. Mirtax (Sandoz Co.) is a widely used generic mirtazapine that was approved by the United States Food and Drug Administration in 2003. In fact, most generics are released following evidence that their pharmacokinetics are similar to those of the original drug, ${ }^{6)}$ and few clinical trials

(c) This is an Open-Access article distributed under the terms of the Creative Commons Attribution Non-Commercial License (http://creativecommons.org/licenses/by-nc/4.0) which permits unrestricted non-commercial use, distribution, and reproduction in any medium, provided the original work is properly cited. 
have been conducted. Because of the similar pharmacokinetic profiles, we presume that the effects of generic mirtazapines are similar to those of branded mirtazapine. However, generic drug sometimes did not show the efficacy as same as branded drug. ${ }^{7)}$ About paroxetine, there are potential differences in efficacy and safety between paroxetine mesylate and paroxetine hydrochloride. ${ }^{8)}$ It also is unknown whether generic mirtazapine is effective or not due to a lack of evidence. Therefore, to evaluate the effects of generic mirtazapine, we conducted a phase 4 clinical trial of Mirtax film-coated tablets. This study simply focused on the Mirtax treatment itself without adopting any control group or switching design from branded medication. However, to our knowledge, this is the first clinical trial to evaluate the efficacy and tolerability of generic mirtazapine.

\section{METHODS}

\section{Study Design}

This was an open-label, uncontrolled, prospective 6-week study to evaluate efficacy and tolerability of Mirtax in MDD patients. It was conducted at 9 centres in Korea from June 2012 to December 2013. Recruitment was accomplished by recommendation from clinicians at the outpatient clinic without any advertisement process. Enrolment criteria were: (1) adult patients over 20 years of age who were diagnosed with MDD according to Diagnostic and Statistical Manual of Mental Disorders, 4th edition, text revision (DSM-IV-TR) criteria, (2) a 17-item Hamilton Depression Rating Scale (HDRS) score of $\geq 14$ at screening, (3) lack of sufficient treatment for the present episode or necessary medication changes due to tolerability problems. Sufficient pharmacological treatment was defined as using appropriate dosage of antidepressant for at least 6 weeks. Appropriate dosages were regarded as $20-40 \mathrm{mg}$ /day of fluoxetine, $20-40 \mathrm{mg} /$ day of paroxetine, $20-40 \mathrm{mg}$ /day of citalopram, $10-20 \mathrm{mg} /$ day of escitalopram, $50-200 \mathrm{mg} /$ day of sertaline, $75-225 \mathrm{mg}$ /day of venlafaxine and 150-300 $\mathrm{mg}$ /day of bupropion. Patients treated with mirtazapine at the point of screening that were also at risk for suicidality or other psychiatric diagnoses were excluded. The screening period was within 2 weeks before baseline measurement; however, when patients had no specific reason for disqualification, screening was permitted following baseline measurement on the same day. Previous antidepressants were stopped after screening. Exclusion criteria were pregnancy or lactation, a medical condition that could interfere with everyday life activities, and psychotic symptoms or previous diagnosis of bioplar disorder or any psychotic disorder. Current primary diagnosis other than MDD, lack of responses during current or a past episode of depression to two or more antidepressant at clinically appropriate doses for a minimum of 6 weeks, and severe suicidal risk were also under the exclusion criteria.

The visit schedule included: screening, baseline, and 1, 2, 4, and 6 weeks. During that period, dosages were started and maintained flexibly at the discretion of the investigator. Any other antidepressants, antipsychotics, mood stabilizers, psychostimulants and buspirone were prohibited; benzodiazepine ( $\leq 3 \mathrm{mg}$ /day of lorazepam-equivalent dosage) and hypnotics were allowed.

The study was conducted according to the Declaration of Helsinki and Good Clinical Practice. Informed consent was obtained from all participants following an extensive explanation of the nature and procedures of the study. The study protocol was approved by the Institutional Review or Ethics Committee of each study site.

\section{Efficacy and Tolerability Assessments}

Efficacy was evaluated using the HDRS, ${ }^{9}$ MontgomeryÅsberg Depression Rating Scale (MADRS), ${ }^{10)}$ the Clinical Global Impressions-Severity Scale (CGI-S) and the Clinical Global Impressions-Improvement Scale (CGI-I). ${ }^{11)}$ The primary efficacy was measured as the mean HDRS change from baseline to week 6. Additional efficacy measures included the mean changes in MADRS, CGI-S scores, and response, remission, and CGI-I $\leq 2$ rates at week 6 . A response was defined as a $\geq 50 \%$ decrease in the HDRS or MADRS score, and remission was defined as an absolute HDRS score $\leq 7$ or MADRS score $\leq 10 .^{12)}$

Tolerability and safety were evaluated with the frequency and severity of AEs, and mean changes in weight, blood pressure, and pulse rate from baseline to week 6 . AEs were filled up in the constructed response questionnaire in the Novartis Adverse Event Report Form.

\section{Statistical Analyses}

Efficacy and safety were analysed in an intent-to-treat (ITT) group, and the last-observation-carried-forward (LOCF) method was applied for endpoint analysis. All patients who received at least one dosage of the study medication were included in the safety analysis.

Data are presented as means \pm standard deviation for quantitative variables and frequencies (percentage) for categorical variables. Quantitative data were analysed by Student's $t$-tests and categorical data were analysed by chi-squared tests. Repeated measures analysis of variance 
(ANOVA) was used to determine the changes in group with adjusting for the time and repeated measures analysis of covariance (ANCOVA) with baseline score as covariate also used to check the different decreases between mild to moderate and severe subgroups. Response and remission rates of each subgroup were also compared. Analyses were performed using SAS software (ver. 9.2; SAS Institute Inc., Cary, NC, USA). Statistical significance was defined as $p<0.05$.

\section{RESULTS}

\section{Patients and Medications}

After screening, a total of 93 patients (70 females, $75.3 \%$ ) entered the study (Table 1). Most patients (61, $65.6 \%$ ) were in their 40 s. Seventy-two patients $(77.4 \%)$ were free from antidepressant treatment at baseline, and 65 patients $(69.9 \%)$ had a prevalence period of less than 1 year. Baseline HDRS, MADRS and CGI-S scores were $25.0 \pm 5.6,30.3 \pm 6.3$ and $5.0 \pm 0.8$, respectively. Mean starting dosage at baseline was $11.5 \pm 6.4 \mathrm{mg} /$ day and mean maintenance dosage at week 4 was $23.1 \pm 9.4 \mathrm{mg} /$ day.

Fourteen patients $(15.1 \%)$ cancelled their consents due to simple change of mind during the first week and were regarded as baseline dropouts. Other dropouts were due to loss to follow-up $(17,18.3 \%)$, adverse events $(7,7.5 \%)$,

Table 1. Baseline demographic and clinical characteristics

\begin{tabular}{lc}
\hline \multicolumn{1}{c}{ Variable } & Data \\
\hline Age (yr) & $50.87 \pm 11.06$ \\
Sex (female) & $70(75.3)$ \\
Married & $78(83.9)$ \\
Prevalence period of depression (mon) & $18.40 \pm 47.35$ \\
Treatment period of depression (mon) & $16.45 \pm 49.06$ \\
Previous history of antidepressant use & $21(22.6)$ \\
Comorbid medical illness & \\
Hypertension & $24(39.3)$ \\
Dyslipidaemia & $11(18.0)$ \\
Diabetes & $9(14.8)$ \\
Renal disorder & $2(3.3)$ \\
Liver disorder & $1(1.6)$ \\
Urinary incontinence & $1(1.6)$ \\
Baseline concomitant medications & \\
Anxiolytics & $59(74.7)$ \\
Hypnotics & $27(34.2)$ \\
$\beta$-blocker & $9(11.4)$ \\
Baseline score of scales & \\
HDRS & $25.08 \pm 5.61$ \\
MADRS & $30.23 \pm 6.34$ \\
CGl-S & $5.0 \pm 0.8$ \\
\hline Val &
\end{tabular}

Values are presented as number (\%) or mean \pm standard deviation.

HDRS, Hamilton Depression Rating Scale; MADRS, MontgomeryÅsberg Depression Rating Scale; CGI-S, Clinical Global ImpressionsSeverity. protocol violations $(4,4.3 \%)$, low compliance $(1,1.1 \%)$, and one quit at the discretion of the investigator (1.1\%). At the end of the trial, 49 patients $(52.7 \%)$ finished the study. ITT analyses included a initially total 93 patients.

\section{Efficacy}

At week 6, the HDRS and MADRS scores were decreased to $11.9 \pm 8.6$ (mean change $-13.1 \pm 8.3, p<0.001$; Fig. 1) and $13.73 \pm 10.40$ (mean change $-16.5 \pm 9.8, p<0.001$; Fig. 2). The percentages of responders were $64.6 \%$ (HDRS) and $60.8 \%$ (MADRS), and those of patients in remission were $35.4 \%$ (HDRS) and $43.0 \%$ (MADRS) (Table 2). CGI-S was decreased to $2.5 \pm 1.3$ (mean change $-2.5 \pm 1.3$, $p<0.001)$ and there were 49 patients $(52.7 \%)$ with a CGI-I score $\leq 2$. Significant decreases in HDRS, MADRS and CGI-S scores were confirmed at week 1 . The response and remission rate also showed great increase during the first week (Table 2).

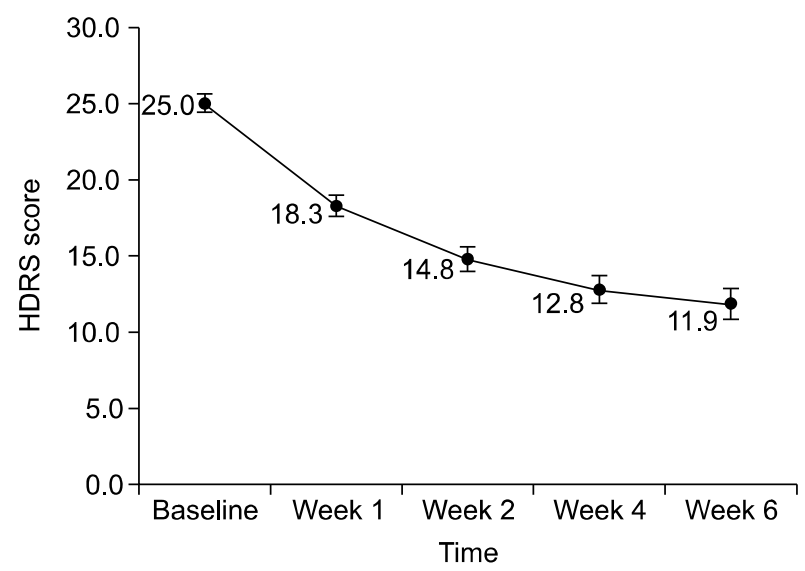

Fig. 1. Change in Hamilton Depression Rating Scale (HDRS) score over 6 weeks.

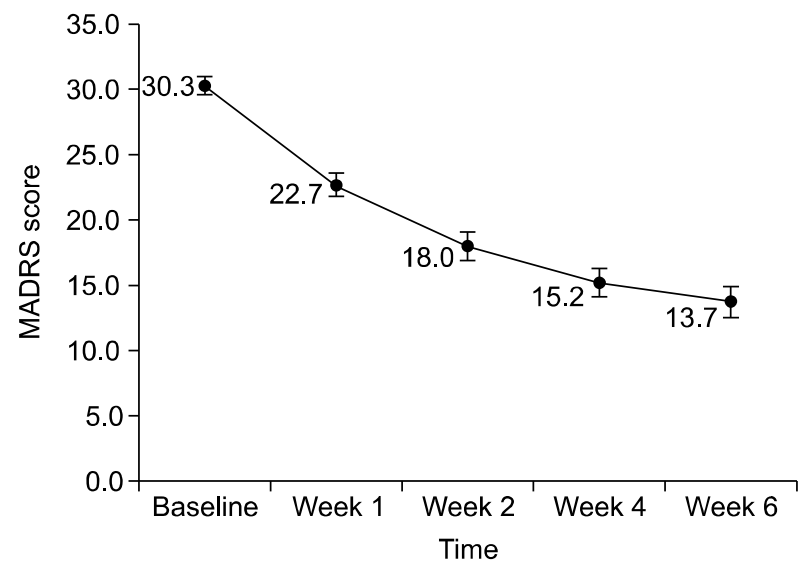

Fig. 2. Change in Montgomery-Åsberg Depression Rating Scale (MADRS) score over 6 weeks. 
Table 2. Percentage of patients classified as responders and remitters by HDRS and MADRS score

\begin{tabular}{|c|c|c|c|c|}
\hline \multirow{2}{*}{ Time } & \multicolumn{2}{|c|}{ HDRS } & \multicolumn{2}{|c|}{ MADRS } \\
\hline & Response rate & Remission rate & Response rate & Remission rate \\
\hline Week 1 & 13.9 & 6.3 & 11.4 & 6.3 \\
\hline Week 2 & 44.3 & 16.5 & 41.8 & 16.5 \\
\hline Week 4 & 50.6 & 29.1 & 54.4 & 31.6 \\
\hline Week 6 & 64.6 & 35.4 & 60.8 & 43.0 \\
\hline
\end{tabular}

HDRS, Hamilton Depression Rating Scale; MADRS, Montgomery-Åsberg Depression Rating Scale.

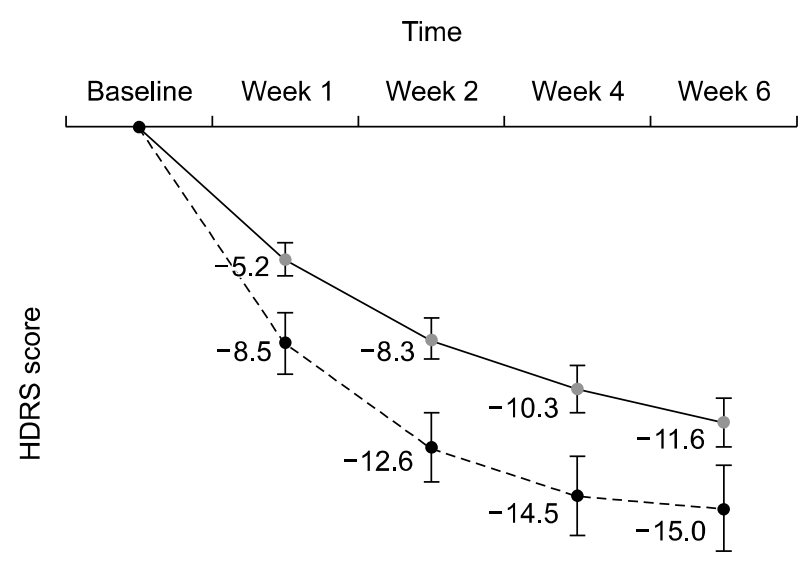

Fig. 3. Difference in decreases of Hamilton Depression Rating Scale (HDRS) score between mild to moderate-to moderate patients and severe patients.

A solid line: patients whose baseline HDRS $\leq 24(n=48)$; a dotted line: patients whose baseline HDRS $>24(n=45)$.

When participants were divided into a mild-to-moderate subgroup (baseline HDRS $\leq 24, n=48$ ) and a severe subgroup (baseline HDRS $>24, n=45$ ), their decreases of HDRS scores from baseline were significantly different between two subgroups ( $p=0.006$, Fig. 3 ). That is, the HDRS scores were more decreased in the severe subgroup than in the mild-to-moderate subgroup. It was much the same for the MADRS scores $(p=0.012)$. However, final response rates at week 6 were not different as $68.2 \%$ (mildto-moderate subgroup) and $60.0 \%$ (severe subgroup, $p=$ 0.125 ), while the remission rates were significantly different as $47.7 \%$ (mild-to-moderate subgroup) and $20.0 \%$ (severe subgroup, $p<0.001$ ).

\section{Adverse Events (AEs) and Safety}

There were a total of 45 AEs in 30 patients, and the total $\mathrm{AE}$ rate was $32.3 \%$. The most frequently reported adverse events were sedation (4.3\%) and constipation (4.3\%), followed by dyspepsia (3.2\%) and thirst (3.2\%) (Table 3). Among them, about two-thirds (30 cases) were mild in severity, and nine cases of adverse events in seven patients $(7.5 \%)$ were related to the drug. Three cases of severe ad-
Table 3. Adverse events during the study period ( $\geq 2 \%$ )

\begin{tabular}{lc}
\hline Adverse event & Total $(n)$ \\
\hline Sedation & 4 \\
Constipation & 4 \\
Dyspepsia & 3 \\
Thirst & 3 \\
Headache & 2 \\
Dry mouth & 2 \\
Nausea & 2 \\
Palpitation & 2 \\
\hline
\end{tabular}

verse events occurred in three patients $(3.2 \%)$, including suicide, unintentional drug intoxication and acute tonsillitis, of which causalities with drug were low.

Twenty-nine patients received no AE treatment, and 23 patients remained in the trial, while 7 patients were withdrawn due to AEs. The AEs related with dropout were sedation (2), headache (2), thirst (1), dyspepsia (1) and suicide ( 1 patient). At the end of the trial, the AEs of 17 patients had improved, while 6 patients were still experiencing them.

Weight increased from $58.8 \pm 10.6$ to $60.3 \pm 9.3 \mathrm{~kg}$ (mean change $0.7 \pm 1.7 \mathrm{~kg}, p=0.004$ ) over 6 weeks. Systolic blood pressure did not change (from $119.5 \pm 5$ to $118.8 \pm 12.0$ $\mathrm{mmHg}$, mean change $-1.27 \pm 7.7 \mathrm{mmHg}, p=0.222$ ), but diastolic blood pressure was decreased from 77.1 \pm 9.4 to $75.9 \pm 8.9$, mean change $-2.4 \pm 8.1 \mathrm{mmHg}, p=0.031)$. Pulse rate did not change (from $74.8 \pm 9.3$ to $73.9 \pm 5.9$ beats $/ \mathrm{min}$, mean change $0.5 \pm 6.4$ beats $/ \mathrm{min}, p=0.584$ ). These findings were not clinically significant.

\section{DISCUSSION}

Although this study employed an open-label and noncomparative design, the results demonstrated the efficacy and tolerability of Mirtax for the treatment of MDD. Significant decreases in the HDRS, MADRS, and CGI-S scores over 6 weeks were confirmed. Rates of CGI-I score $\leq 2$, responders and remitters at week 6 also further support the efficacy of Mirtax. Decreases of HDRS scores 
during 6 weeks were found out more in severe subgroup, while the remission rate at 6 week was above two times more in mild-to-moderate patients group. The incidence of AEs was very low, and most AEs were tolerable and not severe. Drug causality was low, including the severe AEs.

How does it compare with branded mirtazapine? The results from between-groups design are hard to compare with single treatment of this study uniformly. Nevertheless, studies including ours and previous ones revealed generally similar character in efficacy at week 6 . Wheatley et al. ${ }^{13)}$ reported a $63.3 \%$ response rate and a $23.3 \%$ remission rate in a comparison study with fluoxetine. Benkert et $a l .{ }^{14)}$ reported a $58.3 \%$ response rate and a $40.9 \%$ remission rate in a comparison study with paroxetine. Asian people also showed similar efficacy in a comparison study with fluoxetine ( $58 \%$ response rate and $35 \%$ remission rate). ${ }^{15)}$ In two meta-analyses, the pooled response rate for mirtazapine was $67.1 \%,{ }^{16)}$ which is in agreement with our findings $(64.6 \%)$, but the remission rate for mirtazapine was $43.4 \%,{ }^{17)}$ which is somewhat higher than that reported here (35.4\%, standard: HDRS score).

The most distinguished result about branded mirtazapine might have a faster onset of action. Many studies of branded mirtazapine showed efficacy prior to week 2 as compared with SSRIs, which was confirmed by a metaanalysis. ${ }^{18)}$ Another meta-analysis indicated $13 \%$ response rate and $3.4 \%$ remission rate for mirtazapine in week $1{ }^{19)}$ These findings coincided relatively well with our study which demonstrated significant improvements in both HDRS and MADRS, and $13.9 \%$ of response rate and $6.3 \%$ of remission rate at week 1 . In addition, response rate and remission rate markedly rose to $44.3 \%$ and $16.5 \%$, respectively, at week 2 . Considering above findings, Mirtax may have a earlier efficacy similar to branded mirtazapine potentially.

In this study, one characteristic feature was revealed. The degree of decreasing HDRS and MADRS scores was different between severe and mild to moderate patients as having more decrease in severe patients, while the remission rate of a mild-to-moderate subgroup (47.7\%) was above two times more than that of a severe subgroup $(20.0 \%)$. It suggested that mild-to-moderate subgroup seems to have a better therapeutic effect from Mirtax than severe subgroup, even though decreases of HDRS scores from baseline were more in severe subgroup.

About tolerability and safety, only 30 patients (32.3\%) reported spontaneous AEs, which is about half of previous reports $\left(68.1 \%{ }^{14)}\right.$ and $\left.66.4 \%{ }^{20)}\right)$. There was a $47.3 \%$ dropout rate, which was higher than previous studies
$(40 \%){ }^{21,22)}$ We collected AE reports from $84.9 \%$ of participants, with the exception of 14 patients who withdrew their consent by simple change of mind before the first week. Considering these points, this study demonstrated a very low incidence of AEs.

Among AEs, sedation was the most frequent, but its incidence $(4.3 \%)$ was considerably lower than that of the branded drug, which has a sedative rate of up to $54 \%$. ${ }^{23)}$ Also dizziness which had been reported frequently in previous studies $(19.7 \%)^{15)}$ was not reported. These differences might be caused by the relatively low dosage used in this study. This study used a flexible dosage setting based on investigator judgment. As a result, the starting dosage was only $11.5 \pm 6.4 \mathrm{mg} / \mathrm{day}$, and the maintenance dosage was $23.1 \pm 9.4 \mathrm{mg} /$ day, which were relatively low compared with previous studies. The low incidence of AEs may support this notion.

In general, a higher starting dosage appears to increase sedation, although it decreases over time after repeat dosing. ${ }^{24)}$ A higher dosage might have a less marked sedative effect when antihistamine activity is offset after increased noradrenergic transmission. ${ }^{25)}$ The low incidence of sedation and other AEs might be due to the low starting and maintenance dosages. These results may be representative of real-world practice because many clinicians carefully prescribe mirtazapine at less than $15 \mathrm{mg} /$ day from the beginning.

Finally, we confirmed that generic mirtazapine also causes weight gain; however, the degree of weight gain was not clinically significant. Weight gain of $0.7 \pm 1.7 \mathrm{~kg}$ during 6 weeks might occur naturally and regarded safe in the general clinical situation. Considering that two-thirds of AEs were mild and only $7.5 \%$ of AEs were related with drug, Mirtax was found to be well tolerated in real clinical situation.

Our study had a number of limitations including single treatment design and small number of participants. The process also was not as strict as phase 3 clinical trial. There was no formal inter-rater reliability assessment before the study. The average in demographic data was influenced by skewed data and hard to evaluate age and sex effects due to small sample size.

However, this study, as the first clinical trial of generic mirtazapine, demonstrated its efficacy and tolerability over 6 weeks. Additionally, it suggested that Mirtax had fast onset of action similar to branded mirtazapine, and the possibility of differences in side effect profiles.

Even though our initial study might not provide high level of evidence, it showed the importance of investigat- 
ing the generic antidepressants. To address variability between branded and generic drugs, studies into switches from the branded to the generic drug would be more suitable. Further investigation and clinical experience are required to gather those evidences.

\section{Acknowledgments}

This study was supported by Sandoz. All data were collected and analyzed by DreamCIS, the CRO (Contract Research Organization) company.

\section{REFERENCES}

1. Kessler RC, Aguilar-Gaxiola S, Alonso J, Chatterji S, Lee $\mathrm{S}$, Ormel $\mathrm{J}$, et al. The global burden of mental disorders: an update from the WHO World Mental Health (WMH) surveys. Epidemiol Psichiatr Soc 2009;18:23-33.

2. Szegedi A, Schwertfeger N. Mirtazapine: a review of its clinical efficacy and tolerability. Expert Opin Pharmacother 2005;6:631-641.

3. Thompson C. Mirtazapine versus selective serotonin reuptake inhibitors. J Clin Psychiatry 1999;60 Suppl 17:18-22; discussion 46-48.

4. Benkert O, Szegedi A, Philipp M, Kohnen R, Heinrich C, Heukels A, et al. Mirtazapine orally disintegrating tablets versus venlafaxine extended release: a double-blind, randomized multicenter trial comparing the onset of antidepressant response in patients with major depressive disorder. J Clin Psychopharmacol 2006;26:75-78.

5. Benjamin S, Doraiswamy PM. Review of the use of mirtazapine in the treatment of depression. Expert Opin Pharmacother 2011;12:1623-1632.

6. Borgheini G. The bioequivalence and therapeutic efficacy of generic versus brand-name psychoactive drugs. Clin Ther 2003;25:1578-1592.

7. Margolese HC, Wolf Y, Desmarais JE, Beauclair L. Loss of response after switching from brand name to generic formulations: three cases and a discussion of key clinical considerations when switching. Int Clin Psychopharmacol 2010;25:180-182.

8. Pae CU, Misra A, Ham BJ, Han C, Patkar AA, Masand PS. Paroxetine mesylate: comparable to paroxetine hydrochloride? Expert Opin Pharmacother 2010;11:185-193.

9. Williams JB. A structured interview guide for the Hamilton Depression Rating Scale. Arch Gen Psychiatry 1988;45:742747.

10. Williams JB, Kobak KA. Development and reliability of a structured interview guide for the Montgomery Asberg Depression Rating Scale (SIGMA). Br J Psychiatry 2008; 192:52-58.

11. Busner J, Targum SD. The clinical global impressions scale: applying a research tool in clinical practice. Psychiatry
(Edgmont) 2007;4:28-37.

12. Frank E, Prien RF, Jarrett RB, Keller MB, Kupfer DJ, Lavori $\mathrm{PW}$, et al. Conceptualization and rationale for consensus definitions of terms in major depressive disorder. Remission, recovery, relapse, and recurrence. Arch Gen Psychiatry 1991;48:851-855.

13. Wheatley DP, van Moffaert M, Timmerman L, Kremer CM. Mirtazapine: efficacy and tolerability in comparison with fluoxetine in patients with moderate to severe major depressive disorder. Mirtazapine-Fluoxetine Study Group. J Clin Psychiatry 1998;59:306-312.

14. Benkert O, Szegedi A, Kohnen R. Mirtazapine compared with paroxetine in major depression. J Clin Psychiatry 2000; 61:656-663.

15. Hong CJ, Hu WH, Chen CC, Hsiao CC, Tsai SJ, Ruwe FJ. A double-blind, randomized, group-comparative study of the tolerability and efficacy of 6 weeks' treatment with mirtazapine or fluoxetine in depressed Chinese patients. $J$ Clin Psychiatry 2003;64:921-926.

16. Papakostas GI, Homberger CH, Fava M. A meta-analysis of clinical trials comparing mirtazapine with selective serotonin reuptake inhibitors for the treatment of major depressive disorder. J Psychopharmacol 2008;22:843-848.

17. Thase ME, Nierenberg AA, Vrijland P, van Oers HJ, Schutte AJ, Simmons JH. Remission with mirtazapine and selective serotonin reuptake inhibitors: a meta-analysis of individual patient data from 15 controlled trials of acute phase treatment of major depression. Int Clin Psychopharmacol 2010;25:189-198.

18. Watanabe N, Omori IM, Nakagawa A, Cipriani A, Barbui $\mathrm{C}$, Churchill R, et al. Mirtazapine versus other antidepressive agents for depression. Cochrane Database Syst Rev 2011; (12): $C D 006528$

19. Quitkin FM, Taylor BP, Kremer C. Does mirtazapine have a more rapid onset than SSRIs? J Clin Psychiatry 2001;62: 358-361.

20. Leinonen E, Skarstein J, Behnke K, Agren H, Helsdingen JT. Efficacy and tolerability of mirtazapine versus citalopram: a double-blind, randomized study in patients with major depressive disorder. Nordic Antidepressant Study Group. Int Clin Psychopharmacol 1999;14:329-337.

21. Claghorn JL, Lesem MD. A double-blind placebo-controlled study of Org 3770 in depressed outpatients. J Affect Disord 1995:34:165-171.

22. Vartiainen H, Leinonen E. Double-blind study of mirtazapine and placebo in hospitalized patients with major depression. Eur Neuropsychopharmacol 1994;4:145-150.

23. Dewan MJ, Anand VS. Evaluating the tolerability of the newer antidepressants. J Nerv Ment Dis 1999;187:96-101.

24. Kasper S, Praschak-Rieder N, Tauscher J, Wolf R. A risk-benefit assessment of mirtazapine in the treatment of depression. Drug Saf 1997;17:251-264.

25. Iwamoto K, Kawano N, Sasada K, Kohmura K, Yamamoto $\mathrm{M}$, Ebe $\mathrm{K}$, et al. Effects of low-dose mirtazapine on driving performance in healthy volunteers. Hum Psychopharmacol 2013;28:523-528. 